\title{
Discovery of a peculiar dip from GX 301-2
}

\author{
E. Göğüş ${ }^{1}$, I. Kreykenbohm² ${ }^{2}$ and T. M. Belloni ${ }^{3}$ \\ ${ }^{1}$ Sabancı University, Faculty of Engineering and Natural Sciences, Orhanl1- Tuzla, 34956 İstanbul, Turkey \\ e-mail: ersing@sabanciuniv.edu \\ 2 Dr. Karl Remeis-Sternwarte \& ECAP, Universität Erlangen-Nürnberg, Sternwartstr. 7, 96049 Bamberg, Germany \\ 3 INAF - Osservatorio Astronomico di Brera, via E. Bianchi 46, 23807 Merate (LC), Italy
}

Received 11 October 2010 / Accepted 16 November 2010

\section{ABSTRACT}

\begin{abstract}
We present temporal and spectral properties of a unique X-ray dip in GX 301-2 as seen with Rossi X-ray Timing Explorer in May 2010. The X-ray pulsation from the source gradually declined prior to the dip, disappears for one spin cycle during the dip, and is abruptly restored in the spin cycle immediately after the dip. Moreover, the phase-integrated spectrum of the source becomes softer before and during the dip, and it quickly hardens again following the dip. Our findings indicate that the mechanism for pulsations gradually turned off briefly, and the underlying dim and softer emission likely from the accretion column became observable in the brief absence of high-level emission due to wind accretion.
\end{abstract}

Key words. X-rays: stars - pulsars: individual: GX 301-2

\section{Introduction}

GX 301-2 (also known as 4U 1123-62) is a high-mass X-ray binary (HMXB) consisting of a neutron star and the early type companion, Wray 977 (BP Cru). The two objects are in an eccentric orbit $(e=0.462)$ with an orbital period of 41.5 days (Koh et al. 1997). The spin period of the neutron star is $686 \mathrm{~s}$ (Sato et al. 1986). The companion star, Wray 977 is often classified as a B1.2Ia star (Koh et al. 1997), while Kaper et al. (1995) reclassified it as a B1 Iae+ hypergiant at a distance of $5.3 \mathrm{kpc}$. The latter classification makes Wray 977 with a luminosity of $1.3 \times 10^{6} L_{\odot}$ and a mass of at least $48 M_{\odot}$ one of the most luminous and most massive stars in our galaxy. The very high mass loss rate of $\dot{M} \sim 10^{-5} M_{\odot} \mathrm{yr}^{-1}$ gives rise to a very dense but slow $\left(v_{\infty}=400 \mathrm{~km} \mathrm{~s}^{-1}\right)$ stellar wind.

The neutron star in GX 301-2 accretes directly from the dense stellar wind, resulting in a highly variable X-ray light curve that is typical of wind accretion. X-ray luminosity changes of a factor of five within a time frame of an hour are common in GX 301-2 (Rothschild \& Soong 1987). The orbital light curve shows a distinct profile: shortly before periastron, the source exhibits X-ray flaring activity. The X-ray luminosity increases by a factor of $\sim 25$ (Pravdo et al. 1995). Around orbital phase 0.2, the X-ray intensity reaches a minimum as the source passes through the dense inner regions of the stellar wind until it is almost eclipsed by the stellar companion (see e.g. Pravdo \& Ghosh 2001; Leahy 2002, and references therein). Following the minimum, the X-ray luminosity increases again with a weak secondary maximum near apastron. This orbital modulation is explained by Leahy (2002) by the presence of a spirally formed gas stream flowing from the optical companion to the neutron star. The neutron star intercepts the gas stream twice per orbit: shortly before periastron where the neutron star runs along the path of the gas stream, resulting in intense X-ray flaring activity, and also near apastrom giving rise to the secondary peak in the orbital light curve.
Earlier spectral studies of GX 301-2 could describe its X-ray spectrum with a power law modified by an exponential cutoff at higher energies (White et al. 1983). Recent studies, however, were unable to model the spectrum of using simple models, instead they had to employ partial covering models as the spectrum of GX 301-2 is heavily absorbed (see e.g. La Barbera et al. 2005; Kreykenbohm et al. 2004) with $N_{\mathrm{H}}$ ranging from $10^{23}$ up to $2 \times 10^{24} \mathrm{~cm}^{-2}$ (La Barbera et al. 2005). Around $6.4 \mathrm{keV} \mathrm{a}$ very strong iron fluorescence line with an equivalent width from $200 \mathrm{eV}$ up to $1800 \mathrm{eV}$ is present (Leahy et al. 1988). At energies above $20 \mathrm{keV}$, the spectrum is further modified by the presence of a cyclotron resonant scattering feature around $\sim 37 \mathrm{keV}$ (Mihara 1995), which was shown to be strongly pulse-phase dependent in energy and depth (Kreykenbohm et al. 2004).

In this paper we uncover an X-ray dipping behavior that was seen in GX 301-2 for the first time. In the next section, we introduce the 2010 Rossi X-ray Timing Explorer (RXTE) observations of the source. In Sect. 3, we present our detailed temporal and spectral investigations of the RXTE observations on 2010 May 28 during which the source exhibited the X-ray dip, and finally we discuss our results and possible mechanisms that could result in the observed behavior in Sect. 4.

\section{Observations and data processing}

We employ publicly available RXTE observations of GX 301-2 which were performed in 17 pointings between 2010 May 26 and July 22 for a total exposure time of $184.7 \mathrm{ks}$ (Program IDs: 95354 and 95428). Individual pointings had exposures ranging between about 1 and $34 \mathrm{ks}$. Those observations performed under the program ID 95354 lasted over $10 \mathrm{ks}$ each, providing a set of uniquely long and uninterrupted (by the satellite orbit around Earth) X-ray data of GX 301-2.

In our study, we used only the data collected with the Proportional Counter Array (PCA, Jahoda et al. 2006). Typically, one or two proportional counter units (PCU) out of 


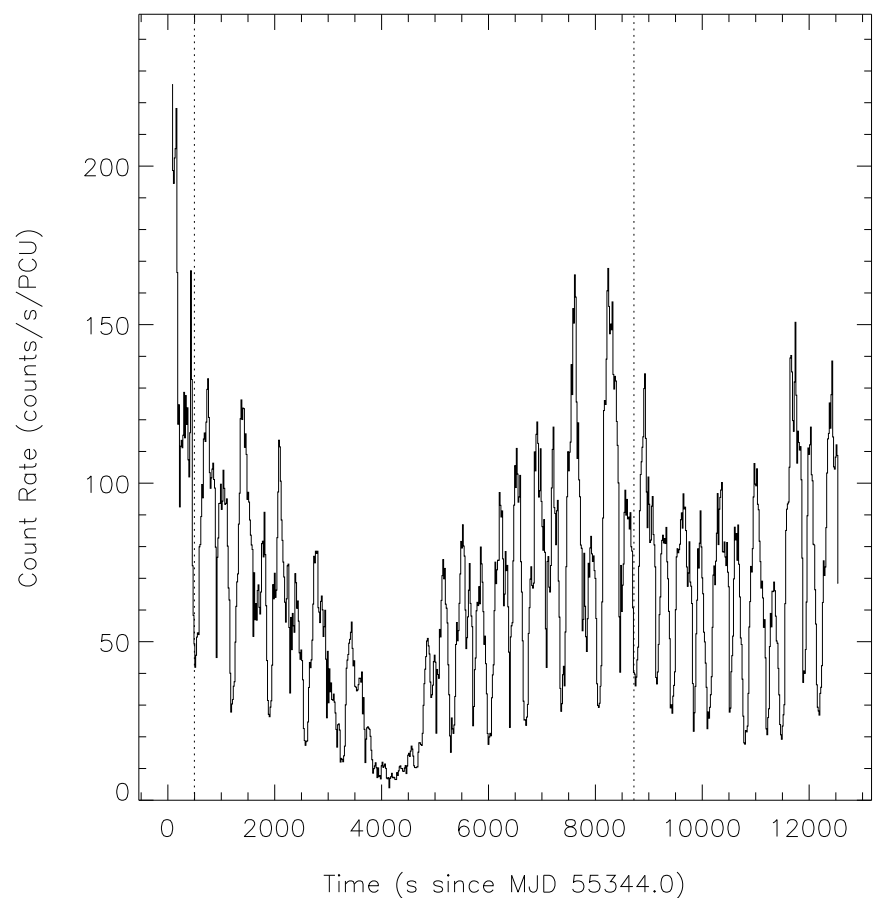

Fig. 1. Light curve of RXTE/PCA observations of GX 301-2 on 2010 May 28 (ObsID: 95354-03-03-00) in the 3-25 keV range. The vertical dotted lines indicate the time interval of detailed temporal and spectral investigations presented in Sect. 3.

five available PCUs were operating during the observations. The combination of operational PCUs varied slightly between observations. We employed all available data for the selected observations for timing studies, and used data collected with PCU2 only for time-resolved spectral investigations to avoid calibration uncertainties (see Sect. 3.2). We processed RXTE data using tools of HEASOFT version 6.9 and performed spectral analysis with XSPEC 12.5.1n (Arnaud 1996).

\section{Data analysis and results}

We uncovered an X-ray dip in the light curve of GX 301-2 as seen on 2010 May 28 for the first time. In Fig. 1, we present the 3-25 keV light curve of the entire exposure span of about $12.4 \mathrm{ks}$ RXTE pointing of the source. X-ray pulses with a period of $686 \mathrm{~s}$ are clearly evident. We find that the X-ray intensity of the source is smoothly decreasing in the episode leading to the dip that lasts about $1000 \mathrm{~s}$ (from MJD 55344.044 to 55344.055). The X-ray intensity of the source gradually increases after the dip and exhibits typical fluctuations as seen from GX 301-2 . Our search in the light curves of all 17 RXTE pointings resulted in no additional dipping episode of the source.

We present below the results of our detailed temporal and spectral investigations of RXTE observations on 2010 May 28 (corresponding to the orbital phase of $\sim 0.7$ using the ephemeris by Doroshenko et al. 2010) to reveal the nature of the dipping behavior.

\subsection{Temporal properties}

In Fig. 2, we show the 3-25 keV band light curves of 12 spin cycles of GX 301-2 in the episode leading up to, during, and following the dip. The vertical axis is plotted on a logarithmic scale to better identify emission structures with low count

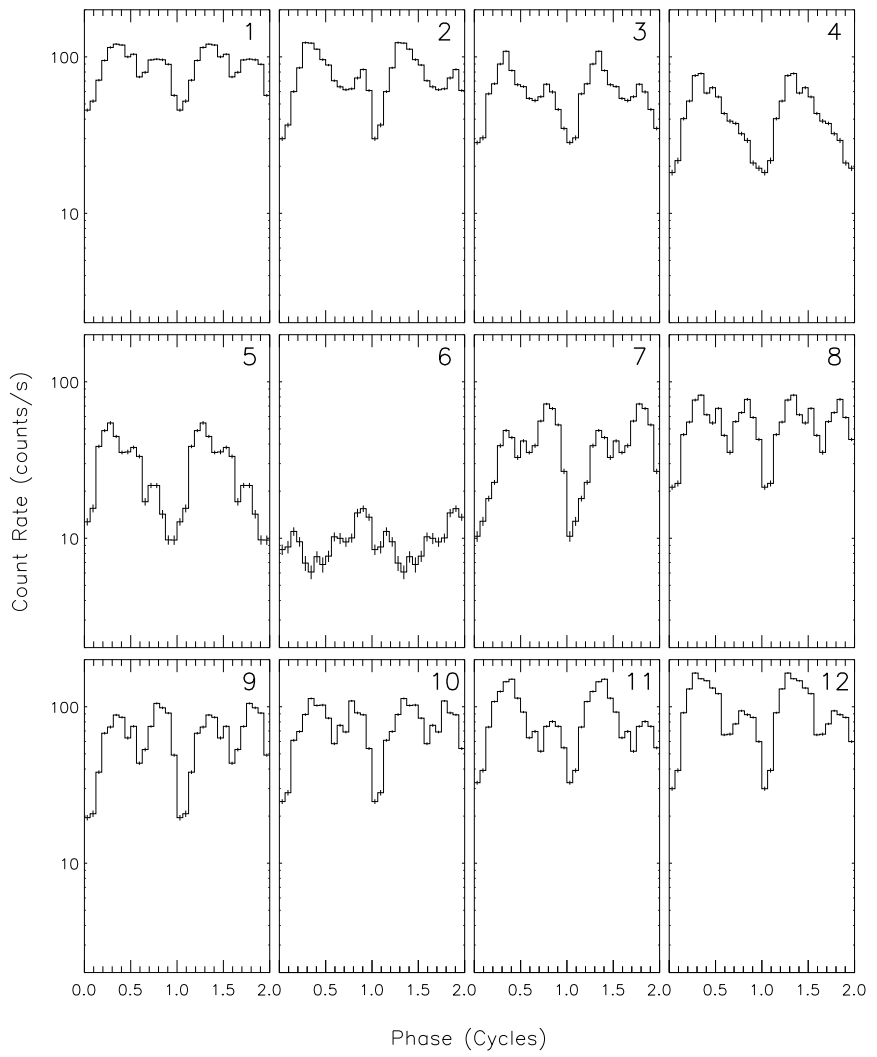

Fig. 2. Spin-phase light curves of GX 301-2 in the 3-25 keV band. Each individual plot is one spin cycle of the pulsar in a continuous time sequence within the time interval indicated in Fig. 1. The $Y$ axes are on a logarithmic scale.

rates. The pulse profile of GX 301-2 is typically characterized by two broad structures; a main pulse and a slightly weaker second peak. We find that in the epoch leading to the dip, while the overall intensity of the X-ray emission decreases, that of the second peak drops faster. Surprisingly, in the dip (cycle number 6), a weak emission structure at the spin phase of the second peak remains, and the main pulse structure from GX 301-2 completely disappears. Following the dip, both emission structures are restored abruptly with the second peak greater than or as strong as the first one for four cycles (number 7-10). After that, the pulse shape of the source resembles its typical long-term appearance again.

We further investigated the evolution of the pulse profile around the dip by means of disentangling the changes in the main pulse and second peak of the pulse profile. For each of the 12 cycles (Fig. 2), we fitted the pulse profile with a model consisting of a constant and two Gaussian components to account for the two peaks. Since the constant level was not constrained well because it was traded off with the Gaussian normalizations, it was fixed at the rate of 8.4 counts/s/PCU, which is the DC level in the dip. To ensure continuity between Phase 1 and 0 , which correspond to the same point, we fitted three versions of each Gaussian shifted by one period ahead and one behind. In other words, each pulse was modeled with three Gaussians separated by a fixed spacing of one period and having the same width and normalization. The fits are homogeneous across the cycles; however, the Gaussian fits to some of the cycles are not perfect $\left(\chi^{2}\right.$ ranging between 8.4 and 49 for 10 degrees of freedom), which is not surprising since the pulse profile of GX 301-2 is more complex than only two Gaussians can describe. Nevertheless, our 


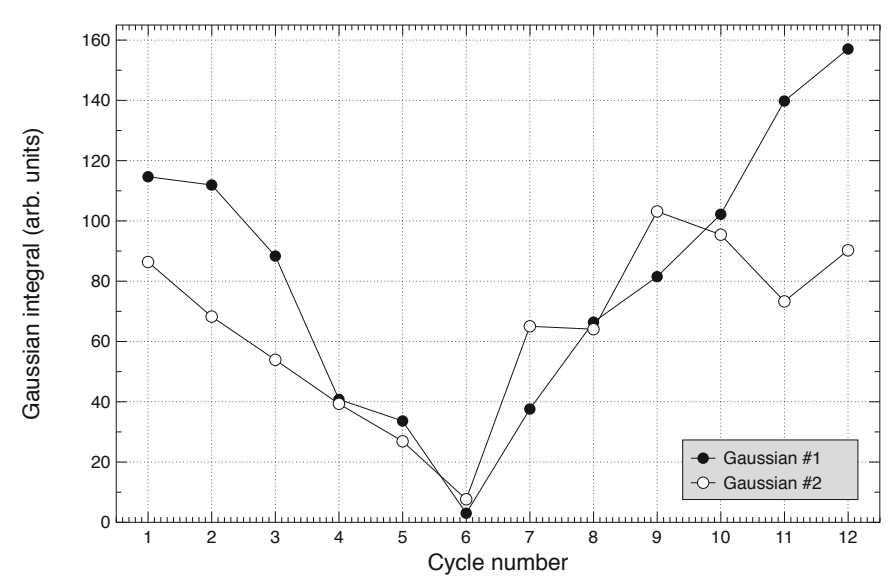

Fig. 3. Evolution of integrated Gaussian functions 1 and 2 that are used to fit the main pulse and the second peak of the pulse profile.

main purpose is to evaluate the time variation of the two broad pulse structures that can be tracked with two Gaussians. We then computed the integral of each Gaussian (1 for the main pulse and 2 for the second peak) in the phase range [0,1], which we show in Fig. 3 as a function of the pulse cycle. It is important to note that both Gaussian integrals gradually decline during the cycles that lead to the dip, and similarly restored gradually following it.

\subsection{Time-resolved spectral properties}

We performed a time-resolved spectral analysis in order to uncover the nature of X-ray emission from GX 301-2 prior to, during, and after the dip. The PCA was operating with two PCUs throughout the time range of interest here. However, only PCU2 was operating over the full time span while PCU3 was active in the first $5.7 \mathrm{ks}$ and PCU4 was operating in the remainder of the observation. Therefore, we accumulated spectral data collected with PCU2 only to avoid any calibration uncertainties. Given that GX 301-2 is a bright X-ray source, we could obtain high signal-to-noise spectra even with one PCU. We accumulated phase-integrated source and background spectra for the 12 time intervals shown in Fig. 2. We modeled X-ray spectra in the 3-25 keV range, where the sensitivity of PCA instrument is the highest (Jahoda et al. 2006).

We first fitted each spectrum individually with an absorbed power law plus a Gaussian model. We find that this simple model fits all 12 spectra adequately well ( $\chi_{v}^{2}$ ranging between 0.66 and $1.23)$. We find that the hydrogen column density varies between $1.3 \times 10^{23}$ and $1.7 \times 10^{23} \mathrm{~cm}^{-2}$ but is consistent with each other within errors. Similarly, the centroid energy and width of the Gaussian function (that accounts for $\mathrm{Fe}$ emission) do not vary across the accumulated spectra. Therefore, we performed a simultaneous fit of all 12 spectra by linking absorption, Gaussian centroid, and width parameters, but allowing power-law index and normalizations of both parameters to vary for all spectra. The resulting best fit yields $N_{\mathrm{H}}=(1.6 \pm 0.2) \times 10^{23} \mathrm{~cm}^{-2}$ with a fit statistics of $\chi^{2} /$ degrees of freedom $=531.4 / 551$. The $N_{\mathrm{H}}$ we obtain here is consistent with what is determined for the orbital phase interval of 0.62-0.65 using BeppoSAX observations (La Barbera et al. 2005). We find that the spectrum of the source becomes softer starting with spin cycle number 4 and the softening trend peaks at the dip (see Fig. 4). The spectral energy distribution is restored back to the pre-dip shape immediately after

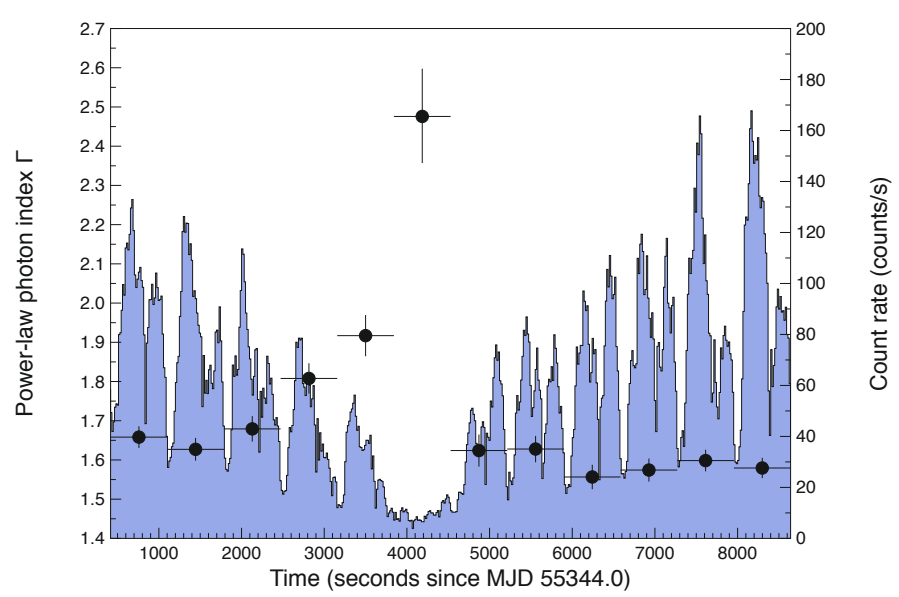

Fig. 4. Power-law indices obtained by spectral modeling (filled circles) and the background subtracted light curve of the source in the $3-25 \mathrm{keV}$ range (blue histograms). The horizontal bars indicate one spin cycle (i.e., $686 \mathrm{~s}$ ) over which each individual spectrum is extracted. The vertical bars are $1 \sigma$ errors in power-law indices.

the dip and remains constant (within errors) in the spin cycles investigated here.

\section{Discussion}

We unveiled intriguing properties of the first observed X-ray dip from GX 301-2. (i) The X-ray pulsation from the source almost completely disappears for one spin cycle only during the dip and comes back to light in the spin cycle immediately after the dip; (ii) The phase-integrated spectrum of the source becomes softer right before and during the dip and it hardens again in the spin cycle immediately after the dip. Both properties point toward the fact that the mechanism for pulsations gradually turned off briefly, therefore, leading to the observed dip in GX 301-2.

Although the observed dip in GX 301-2 is rather unique, other massive wind-accreting X-ray binaries also exhibit a similar dipping behavior, in particular, Vela X-1 $\left(P_{\text {spin }}=283 \mathrm{~s}\right)$. In a long-term light curve taken with INTEGRL/ISGRI of Vela X-1, Kreykenbohm et al. (2008) observed several sudden drops in the count rate. Similar to the dip discussed in this work, Vela X-1was also pulsating normally at a flux level of $\sim 250 \mathrm{mCrab}$, when the pulsations ceased in less than a minute. After about four spin cycles, pulsations went back to normal. During these dips in Vela X-1, the source was undetectable in $20 \mathrm{keV}$ to $40 \mathrm{keV}$ band by INTEGRAL/ISGRI.

Another such interesting dip was observed in a short $R X T E$ observation again of Vela X-1(Kreykenbohm et al. 1999): at the beginning of the observation, Vela X-1was not pulsating when the flux was at a very low level (37 counts/s/PCU in the 3-25 keV compared to 300 counts/s/PCU during normal bright state; see the inset of Fig. 5). Our re-analysis of these data (ObsID: 10141-01-03-00) shows that the source is detected in the PCA, but at a very low flux level and indeed no pulsations are present during this period. We extracted X-ray spectra from the time period when the source was in this dim nonpulsating state and from the bright pulsating state later in the same observation (see Fig. 5). The spectral change between the two states is already evident when we rescale the spectral model of the bright pulsating state to the flux level of the dim phase spectrum: the spectrum of Vela X-1is significantly softer during the dim non-pulsating phase. Therefore, GX 301-2 and Vela X-1 


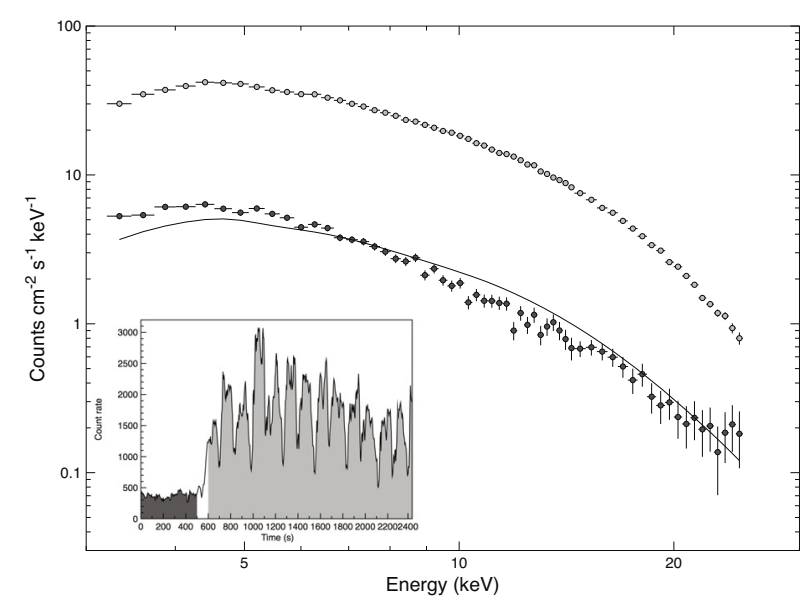

Fig. 5. Comparison of two X-ray spectra of Vela X-1 during the dip (filled circles, extracted from the time segment marked with dark gray in the inset) and in pulsing phase (empty circles, extracted time segment marked with light gray in the inset). The solid line is the rescaled model curve that describes the non-dip spectrum for comparison with the spectrum of the dip.

show the same behavior: they both exhibit short periods of time during which they are very dim, no pulsation is seen, the spectrum is significantly softer and recovery back to pulsing state is abrupt.

Gradual reduction and disappearance of X-ray pulsation in GX 301-2 suggests that X-ray emitting regions of the source were blocked by the wind material (Kretschmar et al. 1999). However, one would expect spectral hardening during the dip as softer X-ray photons would be absorbed. Since we observe significant spectral softening during the gap, it is unlikely that the dip is caused by stellar material moving into the line of sight. GX 301-2 is a source that has exhibited rapid spin-up episodes (Koh et al. 1997). As a result of an extremely rapid spin-up, the infalling material can be expelled via the propeller effect. Cui (1997) reported the disappearance of X-ray pulsations in GX 1+4 and GRO J1744-28 during low-luminosity states and suggested that they are probably caused by the propeller effect.
In the case of the dip in GX 301-2, however, it is not likely to be due to the propeller effect since the system would likely remain in the propeller regime much longer than one spin cycle. Another possibility is that there could be a decrease and brief cessation of matter flowing onto the magnetic poles of the neutron star. In this scenario, the observed low-level emission could be from the underlying polar region - a much larger area than the size of the accretion column, and activated by recent inflow of matter. The low-level emission can easily be blanketed by much brighter emission of accretion. It is still not clear how this picture accommodates the complete disappearance of the pulse lasting only one spin cycle.

Acknowledgements. The authors acknowledge EU FP6 Transfer of Knowledge Project Astrophysics of Neutron Stars (MTKD-CT-2006-042722). T.M.B. acknowledges support from ASI grant I/088/06/0.

\section{References}

Arnaud, K. A. 1996, in Astronomical Data Analysis Software and Systems V, ed. G. Jacoby, \& J. Barnes, ASP Conf. Ser., 101, 17

Belloni, T., Psaltis, D., \& van der Klis, M. 2002, ApJ, 572, 392

Cui, W. 1997, ApJ, 482, L163

Doroshenko, V., Santangelo, A., Suleimanov, V., et al. 2010, A\&A, 515, A10

Jahoda, K., Markwardt, C. B., Radeva, Y., et al. 2006, ApJS, 163, 401

Kaper, L., Lamers, H. J. G. L. M., Ruymaekers, E., van den Heuvel, E. P., \& Zuiderwijk, E. J. 1995, A\&A, 300, 446

Koh, D. T., Bildsten, L., Chakrabarty, D., et al. 1997, ApJ, 479, 933

Kretschmar, P., Wilms, J., Stanbert, R., et al. 1999, ApL \& C, 38, 157

Kreykenbohm, I., Kretschmar, P., Wilms, J., et al. 1999, A\&A, 341, 141

Kreykenbohm, I., Wilms, J., Coburn, W., et al. 2004, A\&A, 427, 975

Kreykenbohm, I., Wilms, J., Kretschmar, P., et al. 2008, A\&A, 492, 511

La Barbera, A., Segreto, A., Santangelo, A., Kreykenbohm, I., \& Orlandini, M. 2005, A\&A, 438, 617

Leahy, D. A. 2002, A\&A, 391, 219

Leahy, D. A., Matsuoka, M., Kawai, N., Koyama, K., \& Makino, F. 1988, PASJ, 40, 197

Mihara, T. 1995, Ph.D. Thesis, RIKEN, Tokyo, Japan

Pravdo, S. H., \& Ghosh, P. 2001, ApJ, 554, 383

Pravdo, S. H., Day, C. S. R., Angelini, L., et al. 1995, ApJ, 454, 872

Rothschild, R. E., \& Soong, Y. 1987, ApJ, 315, 154

Sato, N., Nagase, F., Kawai, N., et al. 1986, ApJ, 304, 241

White, N. E., Swank, J. H., \& Holt, S. S. 1983, ApJ, 270, 711 